\title{
Control of Exciton Fine Structure Splitting in Quantum Dots
}

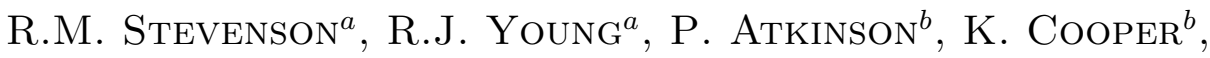 \\ D.A. RitchiE ${ }^{b}$ AND A.J. ShIELDS ${ }^{a}$ \\ ${ }^{a}$ Toshiba Research Europe Limited, 260 Cambridge Science Park \\ Milton Road, Cambridge, CB4 0WE, UK \\ ${ }^{b}$ Cavendish Laboratory, University of Cambridge \\ Madingley Road, Cambridge, CB3 0HE, UK
}

\begin{abstract}
Quantum dots have been identified as potential sources of entangled photon pairs, essential for many applications in quantum information. However, in practice structural properties of the dots result in polarisation splitting of the emission lines, which has prevented the realisation of such a source. Here, we present two techniques developed to control the polarisation splitting in a quantum dot both irreversibly and reversibly. We find that the splitting is strongly dependent upon the emission energy, or lateral confinement, of the quantum dot. Thus by precise control of the InAs deposition thickness, it is possible to produce ensembles from which dots with zero polarisation splitting can be easily selected. Additionally, we demonstrate that by using modest in-plane magnetic fields to partially mix the bright and dark exciton states, the polarisation splitting can be reversibly tuned to zero for most dots of a certain type. Finally, we demonstrate the emission of triggered entangled photon pairs from a quantum dot with approximately zero splitting with fidelity $>70 \%$.
\end{abstract}

PACS numbers: 03.67.Mn, 78.67.Hc, 78.55.Cr

\section{Introduction}

Entangled photon pair sources are key components for applications such as entanglement based quantum key distribution [1, 2], and optical quantum computers [3], for which it is important that no more than one entangled photon pair is generated in a cycle. If multiple pairs are generated, then errors are introduced which severely limits the scalability of the application. The most widely used technique to generate entangled photon pairs is parametric down conversion [4], which produces a probabilistic number of photons pairs per excitation cycle. In contrast, the biexciton decay in a single quantum dot was proposed [5] to provide 
a source of "triggered" entangled photon pairs, so called because it can produce no more than two photons per excitation cycle.

The emission of a pair of photons from a quantum dot is shown schematically in Fig. 1a, and begins with excitation of the biexciton state XX. The biexciton state is spin neutral, along with the ground state. The spin dependent properties of the emission such as polarisation and the associated energy splitting $S$ are therefore determined by the intermediate exciton state $\mathrm{X}$. In ordinary quantum dots, such as depicted by Fig. 1a, structural properties of the dot such as elongation and strain cause in-plane asymmetry of the exciton wave function, which results in the hybridisation and energy splitting of the optically active exciton spin states via the exchange interaction $[6,7]$. The splitting $S$ of the exciton level allows the
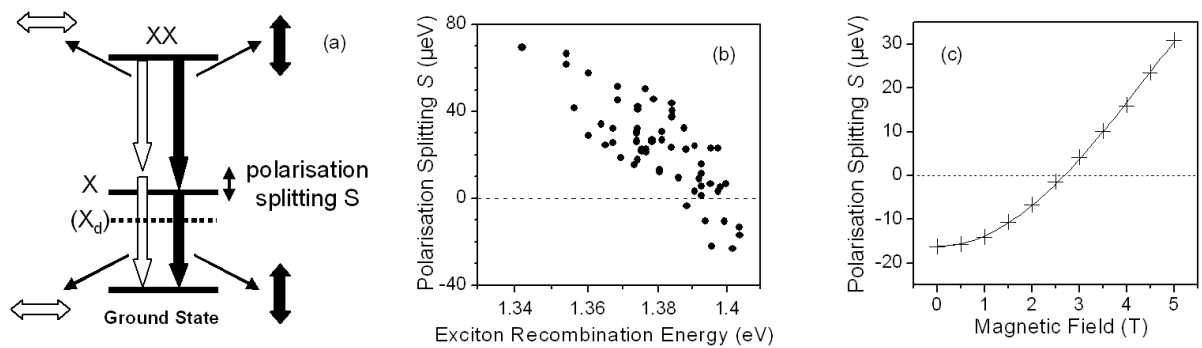

Fig. 1. (a) Schematic showing the radiative decay of the biexciton state (XX) in a typical quantum dot. (b) Polarisation splitting $S$ for many quantum dots as a function of the emission energy. (c) Splitting $S$ as a function of magnetic field of a high emission energy quantum dot with inverted $S$ at $B=0$.

polarisation of each photon to be determined by energy measurements, which represents so-called "which-path" information. This destroys entanglement, resulting in only classically polarisation correlated photon pair emission [7]. The removal of the intermediate exciton level splitting is therefore crucial in order to realise triggered entangled photon pair emission from a dot.

\section{Controlling exciton fine structure splitting by growth}

Previous studies of ensembles of quantum dots have shown that by annealing, it is possible to significantly reduce the polarisation splitting $S$ to below $\sim 10 \mu \mathrm{eV}$, in conjunction with a blue-shift of the dot emission energy [8]. However, further reduction of the splitting is necessary to fall within the homogeneous lifetime, determined from the radiative lifetime to be typically $\sim 1.5 \mu \mathrm{eV}$ in these quantum dots.

Instead of annealing to blue-shift the dot emission energy, here we investigate the effect the emission energy has directly on the splitting. InAs quantum dots were grown by MBE in a GaAs matrix, and single quantum dots were isolated by $2 \mu \mathrm{m}$ apertures in a metal shadow mask. The dots were characterised 
by photoluminescence at $\sim 10 \mathrm{~K}$, with the laser focused and emission collected by the same microscope objective lends. In Fig. 1b, we plot the polarisation splitting $S$ for many individual quantum dots, as a function of the dot emission energies. A clear trend is observed that shows the splitting reduce as the emission energy of the quantum dot increases. However, in contrast to previous experiments, the splitting is crucially reduced close to zero, and even becomes inverted as the dot emission energy increases [9]. Thus, by optimising the growth to produce dots with energy $\sim 1.4 \mathrm{eV}$, it is possible to select dots with splitting less than the homogeneous linewidth required for entanglement. We attribute the relationship between splitting and emission energy as follows [9]. In-plane confinement decreases as the emission energy increases, as verified by the in-plane expansion of the exciton determined by diamagnetic shift measurements [9]. This results in a reduction of the in-plane electron-hole interaction, which reduces the magnitude of the splitting. We note that piezoelectric strain tends to elongate the electron and hole in orthogonal directions in-plane [10], so the change in sign of the splitting could be related to more rapid loss of confinement of one charge carrier.

\section{Controlling exciton fine structure splitting with magnetic field}

It is also possible to reversibly reduce the splitting of some quantum dots by the application of modest in-plane magnetic fields [11]. In addition to the optically active exciton states, quantum dots also feature optically inactive, or dark exciton states. These states $\mathrm{X}_{\mathrm{d}}$, shown schematically in Fig. 1a, are not accessed by radiative biexciton decay, and typically lie a few $100 \mu \mathrm{eV}$ to lower energy of the $\mathrm{X}$ states. Dark and bright states are partially mixed by an in-plane magnetic field, increasing the energies of the each of the predominantly bright states by different amounts, dependent on properties of the dot such as $g$-factors, and exchange energies [11]. We find that dots with inverted splitting at $0 \mathrm{~T}$ typically have the properties required to allow the polarisation splitting to be tuned to $\sim$ zero by magnetic field. Figure 1c plots the splitting for an example dot, which can be tuned between -16 and $31 \mu \mathrm{eV}$ by magnetic fields up to $5 \mathrm{~T}$.

\section{Measurement of triggered entangled photon pairs}

We now determine whether photon pairs emitted from a quantum dot with negligible polarisation splitting are entangled. The sample used was grown by MBE on a GaAs substrate, and included a single self-assembled quantum dot layer, with the thickness of InAs optimised to achieve the desired quantum dot density of $1 \mu \mathrm{m}^{-2}$. AlAs/GaAs distributed Bragg reflectors were grown above (2 repeats) and below (14 repeats) the dot layer to form a planar microcavity, resonant with the optimum quantum dot energy of $1.4 \mathrm{eV}$, which enhances the light collection efficiency from the top of the sample by an order of magnitude [12, 13]. Typically, there is strong background light at these high emission energies from the wetting layer. We suppress this by increasing the growth temperature by $\sim 20^{\circ} \mathrm{C}$, 
to encourage intermixing of the InAs wetting layer with the surrounding GaAs, resulting in a blue-shift of the wetting layer. We find that the relationship between splitting and emission energy is remarkably similar to that of Fig. 1b, despite the blue-shift in the wetting layer, which is expected to affect the in-plane confinement of the quantum dots. By characterising quantum dots that emit around $1.4 \mathrm{eV}$, it was possible to select a dot with approximately zero splitting and low background.

Photoluminescence $(\mathrm{PL})$ was excited at $\sim 10 \mathrm{~K}$, with excitation provided non-resonantly using a $635 \mathrm{~nm}$ laser diode emitting $100 \mathrm{ps}$ pulses with an $80 \mathrm{MHz}$ repetition rate. The properties of photon pairs emitted by a selected quantum dot were analysed by polarisation and time dependent correlations between the XX and $\mathrm{X}$ photons. Emission was isolated by two spectrometers, tuned to the XX and $\mathrm{X}$ emission energies. The insertion of appropriately oriented quarter-wave or half-wave plates preceding each of the spectrometers allows any polarisation measurement basis to be selected. The spectrally filtered XX, and X emission passed through a linear polariser, and polarising beam splitter, respectively, was detected by three silicon avalanche photo-diodes (APDs). The time between detection events on different APDs was measured to determine the second order correlation functions. Finally, the number of counts was integrated over each quantum dot decay cycle.

The probability of detecting coincident photons is dependent on the excitation rate, which drifts and fluctuates during the integration time of our experiments. However, the correlations of the XX detection channel with each of the orthogonally polarised $\mathrm{X}$ detection channels are measured simultaneously with the same excitation conditions, and thus can be compared directly. Additionally, we verify that the time averaged $\mathrm{XX}$ and $\mathrm{X}$ emission from this quantum dot is unpolarised within experimental error, so the number of coincident photon pairs can be normalised relative to the average number of photon pairs separated by at least one cycle, which compensates for the different detection efficiencies of the measurement system. Consequently, to achieve lowest errors we consider mainly the resulting degree of polarisation correlation $C$, which varies between -1 and 1 , where \pm 1 represents perfect polarisation correlation, and 0 represents no polarisation correlation [14].

Figure 2a shows the degree of correlation between $H$ polarised XX and rectilinearly polarised $\mathrm{X}$ photons, as a function of the time delay between the photons, in cycles. For non-zero time delays, $C \sim 0$, indicating that there is no polarisation memory from one cycle to the next. The noise on the data is caused by the statistical errors associated with the finite number of counts, from which the error in $\mathrm{C}$ can be estimated to be typically $<0.05$. At zero time delay, the large peak of $70 \%$ demonstrates a strong polarisation correlation between $H$ polarised excitons and biexcitons in this dot. A similar high degree of polarisation correlation is present for any co-linearly polarised measurement bases for this dot, as 


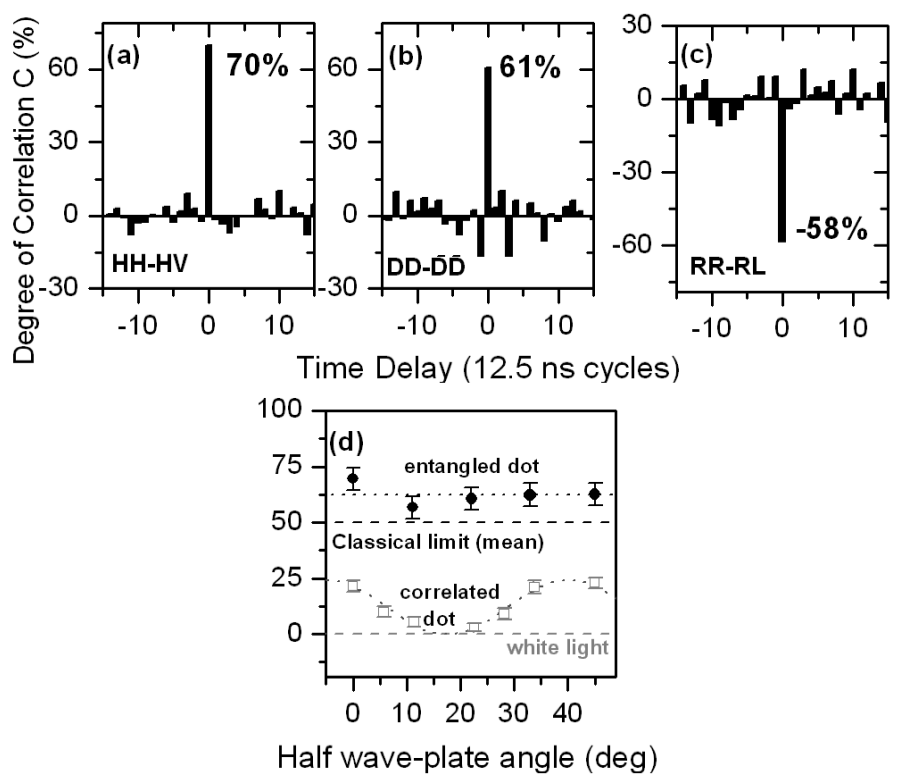

Fig. 2. XX-X polarisation correlations for a dot with polarisation splitting $S \sim 0$. The degree of polarisation correlation $C$ (defined in the text) is plotted as a function of the time delay between the detected photons measured in the rectilinear (a), diagonal (b), and circular bases (c). The degree of correlation is shown in (d) as a function of the rotation of the linear detection basis by a half-wave plate for an entangled dot, and also for a dot with $S>0$ from a sample with stronger background light emission, fitted by a sinusoidal line. The dashed line shows the upper average limit for a classical source, and the grey dashed line shows the average expected for an unpolarised (e.g. white light) source.

shown in Fig. $2 \mathrm{~b}$ for the diagonal measurement basis, and Fig. $2 \mathrm{c}$ for the circular measurement basis.

Figure $2 \mathrm{~d}$ plots the degree of correlation as the function of the angle of a single half-wave plate, placed directly after the microscope objective. It is found that the degree of polarisation correlation $C$ is approximately independent of the half-wave plate angle. This is an expected result for photon pairs being emitted in the entangled $\left(\left|H_{\mathrm{Xx}} H_{\mathrm{X}}\right\rangle+\left|V_{\mathrm{Xx}} V_{\mathrm{X}}\right\rangle\right) / \sqrt{2}$ state, since the linear polarisation measurement of the first photon defines the linear polarisation of the second photon. For classically polarisation correlated photon pairs, the degree of correlation varies sinusoidally with wave-plate angle, as shown for a dot with finite splitting and higher background light contribution [15]. The average linear correlation measured is $62.4 \pm 2.4 \%$ for this dot, which is five standard deviations above the $50 \%$ limit for classical pairs of photons, which proves that the quantum dot emits polarisation entangled photon pairs. 
To fully characterise the two-photon state emitted by the dot, the two-photon density matrix can be constructed from correlation measurements, using quantum state tomography [16], which we adapt for input of the degree of correlation $C$. The measurements pairs required are the combinations of the $V, H, L$, and $D$ biexciton polarisations, with the rectilinear, diagonal, and circular polarised exciton detection bases. The resulting density matrix representing the emission from the quantum dot is shown in Fig. 3, with real and imaginary components shown in (a) and (b), respectively.

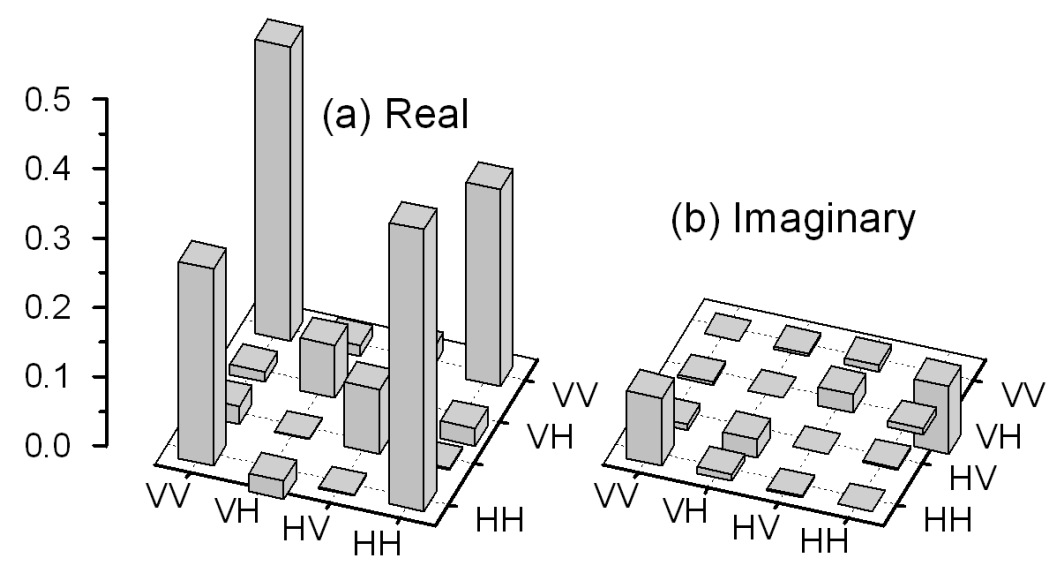

Fig. 3. Real (a) and imaginary (b) components of the density matrix for a quantum dot with polarisation splitting $S<1.5 \mu \mathrm{eV}$. A scale for all elements is shown on the left hand side.

The strong outer diagonal elements in the real matrix demonstrate the high probability that the photon pairs have the same linear polarisation. The inner diagonal elements represent the probability of detecting oppositely linearly polarised photons, which is greatly suppressed over previous measurements, and attributed to roughly equal parts of background light and exciton spin scattering in the dot.

The outer off-diagonal elements in the real matrix are strong, and clear indicators of entanglement. Small imaginary off-diagonal elements are additionally seen, which indicates that there may be a small phase difference between the $|H H\rangle$ and $|V V\rangle$ components of the entangled state. All other elements are close to zero, given the errors associated with the procedure [14].

The measured two-photon density matrix projects onto the expected $\left(\left|H_{\mathrm{XX}} H_{\mathrm{X}}\right\rangle+\left|V_{\mathrm{XX}} V_{\mathrm{X}}\right\rangle\right) / \sqrt{2}$ entangled state with fidelity $0.702 \pm 0.022$. This proves that the photon pairs we detect are entangled, since for pure or mixed classical un-polarised states, the fidelity cannot exceed 0.5. Other tests including the tangle and eigenvalue are also positive for entanglement, by many standard deviations [14]. 


\section{Conclusion}

In conclusion, we present two methods for reducing the polarisation splitting in quantum dots, that otherwise prevents the generation of entangled photon pairs. A relationship between dot emission energy and polarisation splitting is observed, that in turn allows growth to be optimised to produce dots with small, or inverted polarisation splitting. Thus dots can be selected with naturally small splitting, or otherwise can be reduced to zero with an in-plane magnetic field. We directly demonstrate triggered emission of polarisation entangled photon pairs from a single quantum dot with splitting within the homogeneous linewidth. By modifying the growth conditions to blue-shift in the wetting layer, we significantly reduced the background light intensity. The resulting improvements to the degree of polarisation correlation are significant, confirming background light to be a limiting factor to the observed degree of entanglement in degenerate quantum dots. We hope that with further optimisation, the future for an entangled photon LED [17], and associated applications, is very promising.

\section{Acknowledgments}

This work was partially funded by the EU projects SANDiE and QAP, and by the EPSRC.

\section{References}

[1] A.K. Ekert, Phys. Rev. Lett. 67, 661 (1991).

[2] H.-J. Briegel, W. Dür, J.I. Cirac, P. Zoller, Phys. Rev. Lett. 81, 5932 (1998).

[3] E. Knill, R. Laflamme, G.J. Milburn, Nature 409, 46 (2001).

[4] Y.H. Shih, C.O. Alley, Phys. Rev. Lett. 61, 2921 (1988); T.E. Kiess, Y.H. Shih, A.V. Sergienko, C.O. Alley, Phys. Rev. Lett. 71, 3893 (1993).

[5] O. Benson, C. Santori, M. Pelton, Y. Yamamoto, Phys. Rev. Lett. 84, 2513 (2000).

[6] D. Gammon, E.S. Snow, B.V. Shanabrook, D.S. Katzer, D. Park, Phys. Rev. Lett. 76, 3005 (1996); V.D. Kulakovskii, G. Bacher, R. Weigand, T. Kümmell, A. Forchel, E. Borovitskaya, K. Leonardi, D. Hommel, Phys. Rev. Lett. 82, 1780 (1999).

[7] R.M. Stevenson, R.M. Thompson, A.J. Shields, I. Farrer, B.E. Kardynal, D.A. Ritchie, M. Pepper, Phys. Rev. B 66, 081302 (2002); C. Santori, D. Fattal, M. Pelton, G.S. Solomon, Y. Yamamoto, Phys. Rev. B 66, 045308 (2002); S.M. Ulrich, S. Strauf, P. Michler, G. Bacher, A. Forchel, Appl. Phys. Lett. 83, 1848 (2003).

[8] W. Langbein, P. Borri, U. Woggon, V. Stavarache, D. Reuter, A.D. Wieck, Phys. Rev. B 69, 161301(R) (2004); A.I. Tartakovskii, M.N. Makhonin, I.R. Sellers, J. Cahill, A.D. Andreev, D.M. Whittaker, J.P.R. Wells, A.M. Fox, D.J. Mowbray, M.S. Skolnick, K.M. Groom, M.J. Steer, H.Y. Liu, M. Hopkinson, Phys. Rev. B 70, 193303 (2004). 
[9] R.J. Young, R.M. Stevenson, A.J. Shields, P. Atkinson, K. Cooper, D.A. Ritchi, K.M. Groom, A.I. Tartakovskii, M.S. Skolnick, Phys. Rev. B 72, 113305 (2005).

[10] O. Stier, M. Grundmann, D. Bimberg, Phys. Rev. B 59, 5688 (1999).

[11] R.M. Stevenson, R.J. Young, P. See, D.G. Gevaux, K. Cooper, P. Atkinson, I. Farrer, D.A. Ritchie, A.J. Shields, Phys. Rev. B 73, 033306 (2006).

[12] H. Benisty, H. DeNeve, C. Weisbuch, IEEE J. Quantum Electron. 34, 1612 (1998).

[13] A.J. Bennett, D.C. Unitt, P. See, A.J. Shields, P. Atkinson, K. Cooper, D.A. Ritchie, Appl. Phys. Lett. 86, 181102 (2005).

[14] R.J. Young, R.M. Stevenson, P. Atkinson, K. Cooper, D.A. Ritchie, A.J. Shields, New J. Phys. 8, 29 (2006).

[15] R.M. Stevenson, R.J. Young, P. Atkinson, K. Cooper, D.A. Ritchie, A.J. Shields, Nature 439, 179 (2006).

[16] D.F. James, P.G. Kwiat, W.J. Munro, A.G. White, Phys. Rev. A 64, 052312 (2001).

[17] Z. Yuan, B.E. Kardynal, R.M. Stevenson, A.J. Shields, C.J. Lobo, K. Cooper, N.S. Beattie, D.A. Ritchie, M. Pepper, Science 295, 102 (2002). 